\title{
EDITORIAL
}

nature

cell biology

\section{Journal transfer made easy}

Journals in the Nature family are united by the high quality of the papers published, but each journal has a distinct editorial profile to serve a particular, albeit broad, spectrum of the research community. A number of factors can conspire to result in the rejection of a manuscript; prominent among these are the novelty, scope and quality of the data. But it is important to remember that a rejection does not necessarily constitute a criticism of the data; it may simply reflect the editorial judgement of how well it matches the scope of the journal. Often, studies are suitable, in principle, for more than one Nature journal. We realize that casting a manuscript in the shared 'Nature style' and undergoing the elaborate peer review process is a great investment of effort and time; all the more painful if the rejection is ultimately not on the basis of poor data or unfounded conclusions, but because the focus of the study is slightly peripheral for a given journal or because key data for a certain community was missing. Pain can all too easily turn into agony when the dataset in question is of a time-sensitive nature. Given our high rejection rates, we have always made a significant first cut at the editorial level - a process that requires much editorial time and care.

To ensure that potentially excellent manuscripts - usually studies that have already been refereed - do not have to start anew, we have established a system of cross referral between journals: the editor of the rejecting journal may suggest that in their opinion the manuscript is not suitable for their journal, but that another specified Nature journal may be interested, in principle, to pursue the study further. Here it is important to emphasize that this is solely a suggestion, not a guarantee. The editorial staff are not aware of manuscripts under consideration at other journals. However, editors are happy to discuss particular manuscripts in detail with the editors of another Nature journal, if the author so desires; indeed, they are keen to do so when this is clearly in the author's interest. Where referee reports are available, editors can pass these along with the referee identities, and the receiving journal will often be able to make a decision much more rapidly. In our experience this system works well and reduces futile refereeing. We appreciate that our authors may on occasion beg to differ with editorial opinion and may prefer to start the process de novo. This request is always respected and will not prejudice the decision made.

Therefore, we recently developed a system to ease the process of submissions between the Nature journals. This automated manuscript transfer service can be accessed through a link at the bottom of the decision letter. In a nutshell, you can transfer the whole manuscript with cover letter and author affiliation details to another Nature title at the click of a button. Note, however, that referee reports and identities will also be forwarded, if available. If you prefer to use new referees, please submit from scratch. Finally, we have extended this system to the two-dozen specialist academic tiles within NPG (including EMBO Journal and Reports, Oncogene and Cell Death and Differentiation); the only difference here is that referee reports are not forwarded. We very much hope these facilities will aid in finding a suitable home for your study efficiently and painlessly.

\section{The ultimate search engine?}

The need for improved internet searching has grown in direct proportion to the exponential growth of the web. Thus, Google was born to dominate the world of search engines to this day with its deceptively simple searches. The scientific literature is growing apace and the days where a researcher can stay on top of their field by reading across the relevant journals are numbered. We increasingly rely on the highly filtered searches of one of the most efficient engines on the block: PubMed (www.ncbi.nlm.nih.gov/entrez/query.fcgi). The main drawback is that it covers only abstracts and keywords. To address this serious shortcoming, Nature Publishing Group revamped its own search engines (www.nature.com/dynasearch/ app/dynasearch.taf) to provide full access beyond the firewalls and introduced semantic text matching (the section labelled 'more articles like this' on abstract pages). Furthermore, 650 publishers assembled to form CrossRef (www.crossref.org), a non-profit service with the important aim of adding permanence to the web-based literature through a system of digital object identifiers (DOIs; www.doi.org). CrossRef also launched a trial search engine (www.crossref.org/ crossrefsearch.html) last year that permits full content searching of the participating 29 publishers (including NPG) on a Google platform.

In the meantime, Google ran with this idea, launching Google Scholar in November (still in its beta version at http://scholar.google. com). This system promises the Google magic for academic texts. Importantly, the algorithm ranks hits by webpage interconnectivity and weighs them further through inbuilt citation analysis, which is also displayed as a number against a hit. However, although Google citations follow similar trends to the citation standardbearer Thomson ISI, the numbers certainly do not match. Part of the discrepancy may be because Google Scholar has yet to index a significant fraction of the literature. It remains to be seen if the new kid on the block will become an authoritative port of call for coveted bibliometric data. A limitation at present remains a lack of search filters, such as date and article type, and a lack of a solid name/first name link, which will irk all Dr Smiths. With a nod to open-access publishing, Google Scholar throws open links to other versions of a given paper alongside links to the journal, PubMed and PubMedCentral. This feature has some overtones of the challenges to the music industry seen in online music-sharing services such as Napster a few years ago, and it remains to be seen if this will force publishers to rethink copyright. Meanwhile, Elsevier has launched its own search powerhouse, Scopus (www.scopus.com; see Nature 428, 683; 2004) which promises highly structured deep paper-trawling with citation statistics at a price.

Finally, we still want to throw in a word for good-old-fashioned journal browsing: bad search engines force you to browse, but through junk; good search engines circumvent all browsing by leading only to the expected. Nothing beats reading Nature Cell Biology cover to cover. 